ISSN: 2602-8506

Vol. 3, N³., p.241 - 257, julio - septiembre, 2019

\title{
Valoración de costos en la producción lechera del criadero Santa Ana
}

\section{Valuation of costs in the dairy production of Santa Ana Ranch}

Edwin Rafael Oleas Carrillo. ${ }^{1}$, Julio Cesar Benavides Lara. ${ }^{2}$, Alex Arturo Villafuerte

Gavilánez. $^{3}$, \& Fabián Augusto Almeida López. ${ }^{4}$

DOI: https://doi.org/10.33262/visionariodigital.v3i3.694

\begin{abstract}
.
The present investigation, evaluates the production cost of a liter of milk in the Santa Ana hatchery, located in the sector of Cubillines, belonging to the canton Chambo, province of Chimborazo. An economic analysis was carried out, therefore no treatments or repetitions were considered; Only the information was collected through the acquisition of invoices that intervene in the production process. The investigation lasted 60 days. Achieving the following results for the year 2017, the milk production had variations within 12 months, having a high production in the month of November with 15,503.64 liters and a value of 11,003.93 liters in the month of April, being the lowest, which gives an average production of 457.84 liters / milk of the 30 cows in production. Concluding with the determination of the cost of a liter of milk in Santa Ana hatchery of $\$ 0.42$ and having as a margin of profit per liter of milk $\$ 0.03$ and a
\end{abstract}

\footnotetext{
${ }^{1}$ Magister en Dirección de Empresas, Ingeniero Zootecnista, Docente de la Escuela Superior Politécnica de Chimborazo, eoleas@espoch.edu.ec

${ }^{2}$ Magister en Cadenas productivas agroindustriales, Ingeniero Zootecnista, Docente de la Escuela Superior Politécnica de Chimborazo, jbenavides@espoch.edu.ec

${ }^{3}$ Magister en Cadenas productivas agroindustriales, Doctor en Medicina Veterinaria y Zootecnia, Docente de la Escuela Superior Politécnica de Chimborazo, avillafuerte@espoch.edu.ec

${ }^{4}$ Magister en Reproducción Animal, Ingeniero Zootecnista, Docente de la Escuela Superior Politécnica de Chimborazo, fabian.almeida@espoch.edu.ec
} 
benefit / cost of $\$ 1.06$. that is, for each dollar invested the company has a benefit of $\$ 0.06$. We recommend that the improvement plan be applied so that it will be focused on reducing the production costs of the liter of milk, which will improve the profit margin and the cost benefit ratio of the livestock company.

Keywords: Production, Costs, Livestock

\section{Resumen}

La presente investigación, evalúa el costo de producción de un litro de la leche en el criadero Santa Ana, ubicado en el sector de Cubillines, perteneciente al cantón Chambo, provincia de Chimborazo. Realizándose un análisis económico, por lo cual no se consideró tratamientos, ni repeticiones; únicamente se recolectó la información mediante la adquisición de facturas que intervienen en el proceso de producción. La investigación tuvo una duración de 60 días. Logrando los siguientes resultados para el año 2017, la producción de leche tuvo variaciones dentro de los 12 meses, teniendo una alta producción en el mes de noviembre con 15.503,64 litros y un valor de 11.003,93 litros en el mes de abril, siendo el más bajo, lo que da una media de producción de 457,84 litros/leche de las 30 vacas en producción. Concluyendo con la determinación del costo de un litro de leche dentro de criadero Santa Ana de \$ 0,42 y teniendo como un margen de utilidad por litro de leche $\$ 0,03$ y un beneficio/costo de $\$ 1,06$. es decir que por cada dólar invertido la empresa tiene un beneficio de $\$ 0,06$. Recomendamos se aplique el plan de mejoras para que estará enfocado en la reducción de los costos de producción del litro de leche lo que mejorara en el margen de utilidad y la relación beneficio costo de la empresa ganadera.

Palabras claves: Producción, Costos, Ganadería

\section{Introducción.}

Las empresas pecuarias son unidades económicas de producción que combinan los factores internos y externos, mediante técnicas establecidas, con el fin de producir bienes (leche, carne, etc.) que son predestinados al mercado, es por lo tanto, una unidad de control y de toma de decisiones (Garcia, 2009). 
Aproximadamente 150 millones de hogares en todo el mundo trabajan en a la producción de leche. En una gran cantidad de los países en desarrollo, la leche es originada por pequeños agricultores la cual produce ganancias relativamente rápidas y es una fuente importante de ingresos en efectivo (FAO, 2013).

La unidad de producción de leche bovina es considerada un sistema que está compuesto por varios elementos los cuales tenemos: tamaño del hato, nivel de capitalización, nivel de producción, eficiencia reproductiva (días abiertos, presencia de calores, servicios por concepción y presentación del primer estro fértil), nivel de tecnología y comercialización, los cuales interactúan y se relacionan con el ambiente. (Dominique Hervaes, 2000)

Dentro de la explotación pecuaria siempre ha venido suscitándose la movilización de los recursos económicos diariamente, lo cuales son necesarios para poner solución a los problemas existentes. Las formas de estudiar la asignación de estos, es a través del cálculo de costos, ingresos y márgenes, los cuales le permitan al productor evaluar las acciones realizadas y por realizar, además le brinda información para una mejor toma de decisión (Manitt, 2011).

Teniendo en cuenta que los productores del sector lechero se desenvuelven en un ambiente altamente competitivo, es indispensable conocer la realidad técnica de su empresa y su recurso económico.

El sector lácteo es uno de los más importantes dentro de la economía del Ecuador, debido a que plazas de trabajo directas e indirectas en toda su cadena agroalimentaria, comenzando por el ganadero hasta el comerciante de estos productos. De acuerdo a datos del Banco Central del Ecuador, la cadena productiva de la industria láctea en el país genera 1,5 millones de empleos directos e indirectos y su aporte al Producto Interno Bruto (PIB) fue del 8\% en el 2015. (Torres Gutiérrez, 2018)

Actualmente el sector ganadero ha venido a travesando varios cambios en el momento del pago por su producto principal la leche, las empresas industrializadoras han optado por pagar el litro de leche ya no por cantidad, como en tiempos remotos, sino por la calidad con la que 
se produce, por lo que el productor pecuario se ha visto en la necesidad de implementar nuevos sistemas, permitiéndole acercarse cada vez más a lograr los resultados esperados. Para la incorporación de dichas mejoras en la explotación, se requiere que el propietario haga uso de un determinado capital, lo que a su vez da como resultado un aumento en los costos de producción sin tener un valor detallado del precio real por litro de leche bovina.

Es así que se ha visto en la necesidad de constatar en el criadero Santa Ana, cuanto es costo real de producción por litro de leche, tomando en cuenta el sistema de manejo que actualmente se emplea en dicha explotación dedicada a la ganadería, donde determinamos los componentes del costo de producción, costo de producción del litro de leche de la propiedad y la elaboración un plan de mejoras.

Los costos de producción son los gastos necesarios para mantener un proyecto, línea de procesamiento o un equipo en funcionamiento. En una compañía estándar, la diferencia entre el ingreso (por ventas y otras entradas) y el costo de producción indica el beneficio bruto. El costo de producción tiene dos características opuestas, que algunas veces no están bien entendidas en los países en vías de desarrollo. La primera es que para producir bienes uno debe gastar; esto significa generar un costo. La segunda característica es que los costos deberían ser mantenidos tan bajos como sea posible y eliminados los innecesarios (Henao, 2011).

Un plan de mejora es un conjunto de medidas de cambio que se toman en una organización para mejorar su rendimiento, el rendimiento educativo en nuestro caso. Pueden ser de muchos tipos: organizativas, curriculares, etc. En concreto, en nuestro caso las medidas del plan deben tener como objetivo la mejora del rendimiento del alumnado y la disminución del fracaso escolar a la par que se mejora en excelencia. El mejor desarrollo de las competencias escolares deberá ser el eje de cualquier mejora (Rodriguez, 2014).

\section{Metodología}

La presente investigación se desarrollará en el Criadero Santa Ana, sector Cubillines, Cantón

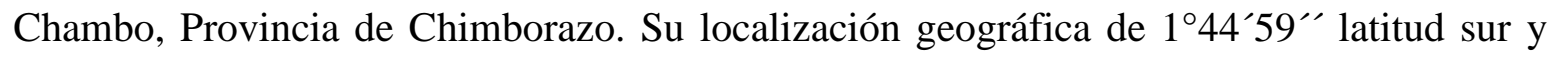


$78^{\circ} 33^{\prime 1} 19^{\prime \prime}$ longitud oeste, a una altitud de 3235 msnm. El tiempo de duración de la esta investigación tendrá una duración de 60 días.

Para el desarrollo de la presente investigación se estableció todos los factores que intervinieron en el proceso de producción internamente de la explotación, se determinaron los costos de producción del litro de leche de acuerdo a los registros obtenidos del criadero Santa Ana. Al relacionarse de una investigación descriptiva y en la cual se hace una evaluación económica de la propiedad, no existe tratamiento alguno, es decir, no existe un esquema del experimento. La información de la actual situación lechera del lugar de estudio se obtendrá con la observación, entrevista con las personas involucradas en la actividad ganadera dentro del lugar. Una vez recolectada la información secundaria, se realizó el formato de encuesta, ya que a partir de esta se aplicará la prueba de encuesta, y así se corrigiera los errores que se puedan dar.

\section{Metodología de evaluación}

Para la evaluación se tomará la información del periodo correspondiente entre enero a diciembre del 2017 y su correspondiente producción de leche del año, se procederá a la clasificación de costos fijos y variables para determinar de manera correcta el costo unitario del litro de leche y establecer su rentabilidad.

\section{Costos fijos}

Los costos fijos son aquellos en los cuales la empresa incurre independientemente del volumen de producción en un período determinado. Éstos, son independientes del nivel de producción, dado que, en el corto plazo, la empresa no tiene la posibilidad de modificar los recursos fijos usados. (Manitt, 2011)

Se recopilará toda la información correspondiente a los costos de los equipos que existen e intervengan en la producción de leche, al igual que las maquinarias y herramientas.

Se tomará en cuenta los roles de pagos a la gente que laboral de forma constante en el sitio de estudio. Para los gastos administrativos se tomarán en cuenta el costo de la compra que se utilizó como por ejemplo los materiales de oficina, cuentas de teléfono, etc. 
Se tomará en cuenta las inversiones fijas y la amortización de cada una de ella como las instalaciones presuntuosas a la actividad, medias sombras corrales, silos de almacenamiento, pisos, etc.- y de los equipos de repartición del alimento (tractores, mixer) que se usan.

\section{Costos variables}

Los costos variables son la parte de los costos totales que varían en el corto plazo según cambia la producción (Henao, 2011); Los costos resultan de añadir insumos variables y que originan aumento en la producción. Para el caso de la agricultura algunos costos variables son semillas, agroquímicos, combustibles, etc. (Manitt, 2011).

Para los costos variables serán tomados los costos de mano de obra ocasional los cuales no entran dentro del rol de pagos normal de la empresa. El gasto en lo relacionados con la alimentación que incluyen forraje verde, alimentos concentrados, sales minerales y ensilajes. Los costos en sanidad incluyen insumos preventivos (vacunas, desinfectantes, etc.), y curativos (antibióticos, antiparasitarios). (Castro, 2017)

\section{Estado de Costos de Producción}

Es el estado financiero que muestra la integración y cuantificación de la materia prima, mano de obra y gastos indirectos que nos ayuda a valorar la producción terminada y transformada para conocer el costo de su fabricación (Gómez Osorio, 2017).

\section{Rentabilidad.}

La rentabilidad es una noción que se aplica a toda acción económica en la que se movilizan unos medios, materiales, humanos y financieros con el fin de obtener unos resultados. En la literatura económica, aunque el término rentabilidad se utiliza de forma muy variada y son muchas las aproximaciones doctrinales que inciden en una $u$ otra faceta de la misma, en sentido general se denomina rentabilidad a la medida del rendimiento que en un determinado periodo de tiempo producen los capitales utilizados en el mismo (Sanchez, 2002).

Para el costo total se sumarán todos los costos fijos y variables en un año de producción.

El costo unitario, se determinará de acuerdo a la producción total de leche en un año de producción. Para lo cual se dividirá el coto total para los litros de leche producidos. 
El indicador Beneficio / costo, se determinará de acuerdo a los egresos totales de producción de leche en un año, dividido para los ingresos. Por concepto de la venta de leche, en el mismo periodo de tiempo.

\section{RESULTADOS Y DISCUSIÓN}

\section{Conformación del hato lechero}

El criadero Santa Ana cuenta con la siguiente conformación del hato lechero por categorías las mismas que se especifican en la tabla 1.

Tabla 1. Conformación del hato lechero.

\begin{tabular}{lcc}
\hline Categoría & Número & Equivalente \% \\
\hline Vacas en producción & 30 & 28,57 \\
Vacas secas & 22 & 20,95 \\
Vacas medias & 23 & 21,9 \\
Vacas fierro & 19 & 18,1 \\
Terneros & 11 & 10,48 \\
\hline Total & 105 & 100 \\
\hline
\end{tabular}

Fuente: Autor (2018).

La propiedad posee una totalidad de 105 cabezas de ganado; los resultados demuestran que el número de animales que posee dicha propiedad no excede la cantidad de sostenibilidad que puede ofrecer la granja. Estos resultados son destacables, al existe un número mayor de vacas en producción $(28,57 \%)$ que es la fuente principal de ingresos. Con una producción promedio de 15 litros de leche diarios por vaca lo que es 3 litros sobre el promedio nacional que se única en 12 litros, en estas datos la producción en el año 2017 fue de 64822,37 litros en el año 2017.

\section{Conformación del costo de producción de leche del criadero Santa Ana}

$\mathrm{Al}$ adquirir los datos de la propiedad, se presentan los siguientes costos fijos y variables presentados en las tablas siguientes: 
ISSN: 2602-8506

Vol. 3, N³., p.241 - 257, julio - septiembre, 2019

\section{Costos fijos:}

Tabla 2. Análisis de los costos de equipos, maquinarias, herramientas del criadero Santa Ana.

\begin{tabular}{lcc}
\hline Descripción & Costo $\$$ & Equivalencia \% \\
\hline Equipos & $1.385,61$ & 18,93 \\
Maquinaria & $4.266,12$ & 58,29 \\
Herramientas & $1.667,41$ & 22,78 \\
\hline Total & $7.319,14$ & 100,00 \\
\hline
\end{tabular}

Fuente: Autor (2018).

El rubro que sobresalen del grupo de datos analizados son las maquinarias que se adquirieron con un costo de $\$ 4.266,12(58,29 \%)$.

El valor invertido de $\$ 7.300 .14$ en equipos maquinarias y herramientas, se considera un tanto elevado, esto puede deberse a diferentes factores como el número de animales en él hato, el tamaño del predio, las necesidades de intensificación, la velocidad de deterioro depende de la calidad de los materiales de los que están compuestos y el recurso económico del propietario.

Tabla 3. Análisis de los costos de mano de obra permanente.

\begin{tabular}{llcr}
\hline Descripción & Cantidad & Costo mensual & Costo anual \\
\hline Vaquero & 3 & 400 & 14.400 \\
Servicios profesionales & 1 & 600 & 7.200 \\
\hline Total & & & 21.600 \\
\hline
\end{tabular}

Fuente: Autor (2018).

Al sumar las variables nos proporcionó un total de egreso de \$21.600. Los valores que el criadero destino a este rubro, está justificado en la contratación de un técnico profesional $(\$ 7.200,00)$; seguido de la contratación de tres vaqueros $(\$ 14.400,00)$. Todos estos pagos que se realizó en una forma permanente, esto se traduce como un gasto fijo que se debe realizar todos los meses del año. Para el pago de este tipo de rubro se debe considerar varios variables 
como los pagos del seguro social, incluyendo los montos por comisiones, bonos mensuales y horas extra.

Tabla 4. Análisis de los costos de gastos administrativos el criadero santa Ana.

\begin{tabular}{lcrr}
\hline Descripción & Cantidad & $\begin{array}{c}\text { Costo } \\
\text { unitario }\end{array}$ & $\begin{array}{c}\text { Precio } \\
\text { total }\end{array}$ \\
\hline Impuesto predial rural & 1 & 11,09 & 11,09 \\
Mantenimiento Incorporación al nuevo catastro rural & 1 & 2,00 & 2,00 \\
Seguro campesino & 1 & 1,29 & 1,29 \\
Empresa eléctrica & 1 & $2.139,70$ & $2.139,7$ \\
Accidentes personales & 12 & 34,86 & 418,3 \\
Libretines de retención & 2 & 9,00 & 18,00 \\
Mantenimiento & 1 & 15,00 & 15,00 \\
Recolección de basura & 1 & 6,00 & 6,00 \\
Teléfono & 12 & 28,96 & 347,50 \\
\hline Total & & & $2.958,91$ \\
\hline
\end{tabular}

Fuente: Autor (2018).

La suma de este gasto fue de \$2.958,91. Este tipo de gastos generales, no están directamente vinculados a una función elemental como la fabricación, la producción o las ventas. Son gastos técnicos necesarios para el funcionamiento básico de una empresa, pero son vitales para el éxito de la organización, siempre y cuando estén destinados a aumentar la eficiencia de una organización. Los gastos administrativos, no se los puede capitalizar, es decir, se los debe gastar en un determinado periodo de tiempo y se debe priorizar como un gasto importante en el balance general.

\section{Costos variables}

Para determinar los costos variables del criadero Santa Ana, se analizó los siguientes componentes:

Tabla 5. Análisis de los costos de la mano de obra ocasional del criadero Santa Ana.

\begin{tabular}{llccc}
\hline Descripción & Cant & $\begin{array}{c}\text { Precio } \\
\text { unitario }\end{array}$ & $\begin{array}{c}\text { No. } \\
\text { Contratos/Año }\end{array}$ & $\begin{array}{c}\text { Costo } \\
\text { anual }\end{array}$ \\
\hline Técnico en mantenimiento ordeñadora mecánica & 1 & 100 & 2 & 200 \\
Chequeos ginecológicos & 1 & 50 & 12 & 600 \\
Jornaleros & 8 & 15 & 12 & 1.440 \\
\hline Total & & & & 2.240 \\
\hline
\end{tabular}

Fuente: Autor (2018). 
El total del rubro de la mano de obra ocasional fue de \$2240,00 al año, esto se debe al manejo y cuidado de maquinarias, al número de animales que posee dentro del hato, la extensión de terreno apta para la agricultura, las prácticas de conservación de forraje etc. en los cuales se requieren jornaleros ocasionales según la necesidad para él trabajo.

Tabla 6. Análisis de los costos de alimentación del criadero Santa Ana.

\begin{tabular}{lcc}
\hline Descripción & Costo \$ & Equivalencia \% \\
\hline Fertilizantes & $9.240,10$ & 41,44 \\
Semillas & $9.444,00$ & 42,35 \\
Suplementos Alimenticios & $3.615,00$ & 16,21 \\
\hline Total & $22.299,10$ & 100,00 \\
\hline
\end{tabular}

Fuente: Autor (2018).

Al analizar el rubro de alimentación, se puede observar el mayor gasto fue en la compra de fertilizantes dando un valor de $\$ 9.240,10$ (41,44\%); seguido por las compras realizadas en semillas $\$ 9.444(42,35 \%)$; y el valor más bajo dentro del grupo de datos son los suplementos alimenticios (balanceados, sales minerales etc.) que se le brindan con una cifra de $\$ 3.615$ $(16,21 \%)$.El total que se gastó en lo que corresponde alimentación fue de $\$ 22.299,10$, esto se debe al número de animales que posee la propiedad, el tipo de balanceado que usa, y principalmente del manejo de la ganadería.

En una explotación bovina alimentación es el rubro más importante representando un costo el 70\% aproximadamente. De acuerdo a Manitt, M.(2011) los principales componentes de este rubro son pasturas, suplementos, etc. En el caso de las pasturas, éstas pueden ser perennes o anuales. Para el cálculo del costo de las últimas se procede de la misma forma que para un cultivo de cosecha. En el caso de las perennes se deberá incluir la amortización y el mantenimiento. 
Vol. 3, N³., p.241 - 257, julio - septiembre, 2019

Tabla 7. Análisis de los costos por sanidad del criadero Santa Ana.

\begin{tabular}{lrr}
\hline Descripción & Costo \$ & Equivalencia \% \\
\hline Antibióticos & 469,11 & 16,2 \\
Desparasitantes & 112,20 & 3,87 \\
Hormonas & 103,58 & 3,58 \\
Vacunas & 515,86 & 17,81 \\
Vitaminas & 206,44 & 7,13 \\
Desinfectantes & 421,13 & 14,54 \\
Otros & 359,00 & 12,4 \\
Varios & 708,71 & 24,47 \\
\hline Total & $2.896,03$ & 100,00 \\
\hline
\end{tabular}

Fuente: Autor (2018)

Entre los datos obtenidos y analizados los valores de mayor relevancia que se reporto fue en gastos varios (jeringas, overoles, agujas, detergentes alcalinos, papel para la limpieza de ubres etc.) con un valor de $\$ 708,71(24,47 \%)$; seguido por gastos en vacunas con una cifra de $\$ 515,86(17,81 \%)$, en antibióticos $\$ 469,11(16,20 \%)$, esto se debe al manejo de hato lechero, y las enfermedades presentes en el área, además del calendario sanitario que esté utilizando le finca.

Para Dominique H., et al. (2000) las cifras que se les asigna al rubro son exclusivamente del precio de los medicamentos sin calcular el costo por la atención del veterinario, ya que es rara la ocasión el uso de dicho profesional sea en casos de emergencia del veterinario particular tendrá que dirigirse a la propiedad. La diferencia de costo por cada atención veterinaria en muy pequeña.

Tabla 8. Análisis de los costos de reproducción del criadero Santa Ana.

\begin{tabular}{lrr}
\hline Descripción & Costo $\$$ & Equivalencia \% \\
\hline Hormonas & $1.149,95$ & 32,48 \\
Pajuelas & $2.280,00$ & 64,40 \\
Varios & 110,66 & 3,13 \\
\hline Total & 3540,61 & 100,00
\end{tabular}

Fuente: Autor (2018). 
ISSN: 2602-8506

Vol. 3, N³., p.241 - 257, julio - septiembre, 2019

El resultado de \$ 3540,61 para reproducción se deben al manejo propio de cada una de las ganaderías, el método reproductivo que usa (inseminación artificial, lavado de embriones etc.), la cantidad de compra de pajuelas, el tipo de conservación de las pajuelas y al número total de animales que se operan en la explotación.

Gasque (2010), manifiesta que la reproducción es considerada a la actividad más indispensable, ya que mediante la aplicación de técnicas determinadas el hato podrá renovar sus animales

\section{Análisis de la producción de leche}

La producción de leche durante los meses de enero hasta diciembre del 2017, del criadero Santa Ana, fueron aproximadamente de 164.822,37 litros, con una desviación estándar de $\pm 111,87$.

Tabla 9. Análisis de la producción de leche del criadero Santa Ana.

\begin{tabular}{lll}
\hline Mes/día & $\begin{array}{l}\text { Pdn mensual } \\
\text { Litros/ leche }\end{array}$ & $\begin{array}{l}\text { Equivalencia } \\
\text { \% }\end{array}$ \\
\hline Enero & $14.684,78$ & 8,91 \\
Febrero & $12.455,90$ & 7,56 \\
Marzo & $13.268,69$ & 8,05 \\
Abril & $11.003,93$ & 6,68 \\
Mayo & $14.567,60$ & 8,84 \\
Junio & $12.085,10$ & 7,33 \\
Julio & $14.156,28$ & 8,59 \\
Agosto & $15.446,96$ & 9,37 \\
Septiembre & $14.644,98$ & 8,89 \\
Octubre & $15.503,64$ & 9,41 \\
Noviembre & $13.647,43$ & 8,28 \\
Diciembre & $13.357,07$ & 8,10 \\
\hline Total & $164.822,37$ & 100,00
\end{tabular}

Fuente: Autor (2018). 
Existe un margen considerable de diferencia entre los meses de enero y diciembre esto puede estar influenciado por factores interno y externo, como el tiempo de lactancia, condición corporal, el alimento y tipo de concentrado que le proporciona, el manejo de las enfermedades y parásitos.

\section{Evaluación Económica}

En el criadero Santa Ana su obtuvo un indicador beneficio costo de un valor $\$ 1,06$; lo que simboliza que por cada dólar invertido se logra una ganancia de $\$ 0,06$, lo cual indica que es media considerando los problemas por los que está atravesando la economía del país.

Tabla 10. Costo de producir un litro de leche.

\begin{tabular}{lr}
\hline Rubro & Valor total \\
\hline Ingreso & $74.170,07$ \\
Egresos & $69.974,79$ \\
Producción, leche/año & $164.822,37$ \\
Costo producir, litro de leche & 0,42 \\
Utilidad, litro leche & 0,03 \\
Beneficio costo & 1,06 \\
Punto de equilibrio & $66.966,25$
\end{tabular}

Fuente: Autor (2018).

El punto de equilibrio del criadero Santa Ana está en 66.966,25, este valor representa la convergencia entre los costos variables y costos fijos, donde se cubren los mismos sin generar un beneficio, es decir el beneficios es igual a cero (y no gana dinero, pero tampoco se pierde); esto se traduce en que anualmente una vez cubierta este valor existirán ganancias y un valor menor nos dará pérdidas económicas para la explotación.

Al determinar los rubros de costo fijo y costo variables durante el año 2017 se procedió a determinar los litros de leche los cuales fueron $\$ 164.822,37$; concluyendo que el costo para producir un litro de leche en el criadero Santa Ana tenía un valor de 0,42 centavos de dólar 
y el costo promedio de venta a nivel de finca para el presente año 2017 fue de 0,45 centavos de dólar lo que simboliza un ingreso de \$74.170,07 de dólares americanos.

La rentabilidad es una noción que se aplica a toda acción económica en la que se movilizan unos medios, materiales, humanos y financieros con el fin de obtener unos resultados (Sánchez, 2002, pp.13).

\section{Conclusiones.}

El componente alimentación del ganado del criadero Santa Ana es el mas alto llegando a \$ 22.299,00 que representa un 31,87\% , seguido de la mano de obra permanente que alcanza los \$21.600,00 que representa el 30,84\%, y en tercer lugar está la reproducción con (Castro, 2017)\$3.540,51 lo que representa un 5,06\%. Por lo que estos valores son importantes para tratar de disminuir el costo de producción.

En el criadero Santa Ana para el año 2017 el costo de producción de un litro de leche asciende a \$ 0,42. En una extensión de terreno de 55 hectáreas, con 30 vacas en producción que mantiene un promedio de 15 litros diarios y aun precio de venta de $\$ 0,45$ deja una utilidad de $\$ 0.03$ por cada litro de leche producido, y con un punto de equilibrio de $\$ 66.966,25$ es decir 148.814 litros de leche al año.

$\mathrm{Al}$ realizar el plan de mejoras del criadero Santa Ana, se enmarco los principales problemas que posee dicha propiedad dentro del tema reproductivo, alimentación, sanidad etc. Es necesario iniciar por la mejora de los pastos, de esta manera mejoraremos la calidad de la alimentación que tiene las vacas, e incrementar la producción de leche, mejorándola rentabilidad en el Criadero Santa Ana.

\section{Referencias bibliográficas.}

Alonso, P. Meléndez G. Y Espinosa, O. 2004. Participación porcentual por insumo en los costos de producción láctea mensual. Buenos Aires, Argentina. 
Vol. 3, N³., p.241 - 257, julio - septiembre, 2019

Castro, L. A. G., Ledesma, L. M., \& Arizala, J. A. (2017). Producción, calidad de leche y beneficio: costo de suplementar vacas Holstein con Tithonia diversifolia. Agronomía Mesoamericana, 357-370.

Centro de la Indstria Láctea del Ecuador . (2015). La leche del Ecuador. Obtenido de http://www.pichincha.gob.ec/phocadownload/publicaciones/la_leche_del_ecuador.p df

Comisión de Normas Sanitarias para los Animales Terrestres. (2014). BIENESTAR ANIMAL Y SISTEMAS DE PRODUCCION DE VACAS LECHERAS. Obtenido de http://www.oie.int/fileadmin/Home/esp/Internationa_Standard_Setting/docs/pdf/E_ TAHSC_Feb_2014_Parte_B.pdf

Dirección de Educación Agraria . (2010). MANUAL DE FORRAJES. Buenos Aires.

Dominguez Juan et. al. (2010). Contabilidad y Finanzas. Obtenido de https://www.eoi.es/es/file/17084/download?token=oK426xIP

FAO. (2012). RESUMENES DE LOS PRODUCTOS BASICOS . Obtenido de http://www.fao.org/3/a-i4738s/i4738s07.pdf

FAO. (2013). Producción lechera. Obtenido de http://www.fao.org/agriculture/dairygateway/produccion-lechera/es/\#.WbML07KLTIU

Garcia, A. (2009). Teoria de la economica de la ganaderia . Obtenido de http://www.uco.es/zootecniaygestion/img/pictorex/14_13_06_Teoria_economica.pd $\mathrm{f}$

Gasque et al., R. (2001). Zootecnia en Bovinos Productores de Leche. Mexico.

Gasque, R. (2010). Enciclopedia Bovina. Enciclopedia Bovina UNAM. México.

Gómez Osorio, L. M., Posada Ochoa, S. L., Olivera Ángel, M., Rosero Noguera, R., \& Aguirre Martínez, P. (2017). Análisis de rentabilidad de la producción de leche de acuerdo con la variación de la fuente de carbohidrato utilizada en el suplemento de vacas holstein. Revista de Medicina Veterinaria, (34), 9-22.

Henao, D. (2011). Costos de produccion de un litro de leche . Obtenido de http://repository.lasallista.edu.co/dspace/bitstream/10567/389/1/Costos_producci\% C3\%B3n_litro_leche.pdf

Herrera, J. (2007). Sistema de produccion de leche en granjas bovinas familiares. Obtenido de http://www.sagarpa.gob.mx/desarrolloRural/Documents/fichasaapt/Sistema\%20de 
\%20producci\%C3\%B3n\%20de\%20leche\%20en\%20granjas\%20bovinas\%20familia res.pdf

Herve Dominique , Mario Morodias . (2000). Como operativizar costos de produccion de leche. Obtenido de http://horizon.documentation.ird.fr/exldoc/pleins_textes/pleins_textes_6/b_fdi_35-36/41168.pdf

Instituto de promoción de exportaciones e inversiones. (2014). Obtenido de http://www.proecuador.gob.ec/wpcontent/uploads/2016/07/proec_psi2016_lacteos.pdf

Magariños, O. (2000). Produccion higenica de la leche cruda . Guatemala: Produccion y servicios incorporados S.A.

Manitt, M. (2011). Costos y Márgenes de la empresa agropecuaria . Obtenido de http://www.ciap.org.ar/ciap/Sitio/Archivos/Costos,\%20I...pdf

Manuel Camiruaga et al. (2010). Razas bovinas de leche y sus caracteristicas. Obtenido de http://www7.uc.cl/sw_educ/prodanim/mamif/siii8.htm

Rodriguez, C. (2014). Evaluacion Economica . Obtenido de http://www.dhl.hegoa.ehu.es/iedl/Materiales/19_Evaluacion_economica.pdf

Sanchez, J. (2002). Analisis de rentabilidad de la empresa . Obtenido de http://www.5campus.com/leccion/anarenta

Torres Gutiérrez, X. E. (2018). Estudio de la producción de la industria láctea del cantón Cayambe en el período 2009-2015(Master's thesis, Universidad Andina Simón Bolívar, Sede Ecuador).

Usuño, B. (2018). Evaluacion economica de la hacienda agrupan. Riobamba.

Zavala, J. (2005). Aspectos nutricionles y tecnologicos de la leche . Obtenido de http://www2.congreso.gob.pe/sicr/cendocbib/con3_uibd.nsf/7AE7E7AB111562710 525797D00789424/\$FILE/Aspectosnutricionalesytecnol\%C3\%B3gicosdelaleche.pd $\mathrm{f}$

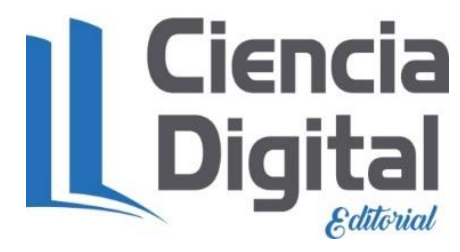




\section{PARA CITAR EL ARTÍCULO INDEXADO.}

Oleas Carrillo, E., Benavides Lara, J., Villafuerte Gavilánez, A., \& Almeida López, F. (2019). Valoración de costos en la producción lechera del criadero Santa Ana. Visionario Digital, 3(3), 241-257. https://doi.org/10.33262/visionariodigital.v3i3.694



El artículo que se publica es de exclusiva responsabilidad de los autores y no necesariamente reflejan el pensamiento de la Revista Visionario Digital.

El artículo queda en propiedad de la revista y, por tanto, su publicación parcial y/o total en otro medio tiene que ser autorizado por el director de la Revista Visionario Digital.
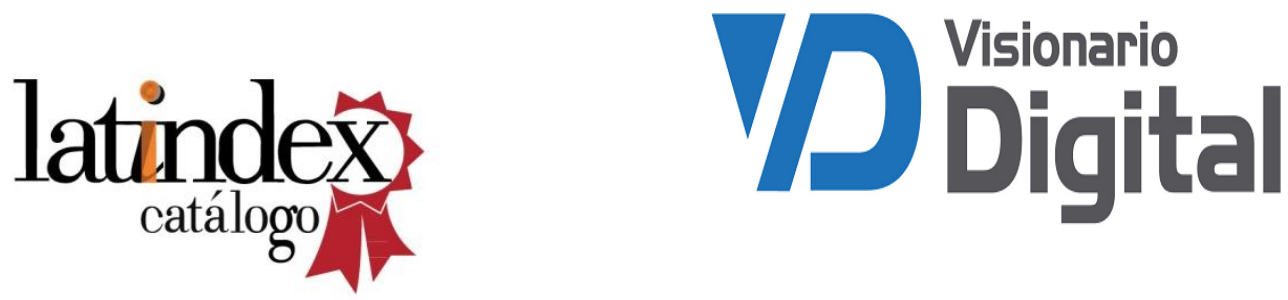